\title{
Is cIMP a second messenger with functions opposite to those of cGMP?
}

\author{
Roland Seifert
}

Received: 16 June 2014 / Accepted: 18 June 2014 / Published online: 15 July 2014

(C) Springer-Verlag Berlin Heidelberg 2014

There is renewed interest in the potential role of noncanonical 3',5'-cyclic nucleoside monophosphates (cNMPs) as second messengers (Akimoto et al. 2014; Chen et al. 2014; Bähre et al. 2014). Inosine 3',5'-cyclic monophosphosphate (cIMP) consists of the base hypoxanthine, the ribosyl moiety and a cyclic phosphate ester linking the $3^{\prime}$ - and 5'-O-ribosyl groups (Fig. 1). cIMP differs chemically from the well-established second messenger guanosine 3',5'-cyclic monophosphate (cGMP) (Schlossmann and Schinner 2012; Levy 2013) by the absence of the amino group at the 2 -position of the nucleobase (Fig. 1). The loss of the amino group results in a reduction in affinity of cIMP for the cGMP effector cGMPdependent protein kinase $\mathrm{I} \alpha(\mathrm{PKGI} \alpha)$ by more than two orders of magnitude (Wolter et al. 2011) (Table 1).

cIMP is generated by highly purified soluble guanylyl cyclase (sGC) in an NO-dependent manner (Beste et al. 2012) and by recombinant particulate guanylyl cyclase (pGC-A) in an atrial natriuretic peptide-dependent manner (Beste et al. 2013), inosine 5'-triphosphate (ITP) being the substrate. The properties of ITP as sGC and pGC substrate compared to the cognate substrate guanosine 5'-triphosphate (GTP) critically depend on the specific experimental conditions (Table 2). At sGC, ITP reaches a higher $V_{\max }$ than GTP in the presence of $\mathrm{Mg}^{2+}$, whereas the opposite is true for the presence of $\mathrm{Mn}^{2+}$. The $K_{\mathrm{M}}$ values of sGC for ITP and GTP are also differentially regulated by $\mathrm{Mg}^{2+}$ and $\mathrm{Mn}^{2+}$. In the presence of $\mathrm{Mn}^{2+}$, sGC exhibits biphasic kinetics for both GTP and ITP (Beste et al. 2012), indicative for the presence of an allosteric regulatory site for ITP and GTP in this enzyme (Seifert and Beste 2012). In contrast to sGC, GTP reaches a higher $V_{\max }$ at pGC-A than ITP with $\mathrm{Mg}^{2+}$, whereas the

R. Seifert $(\bowtie)$

Institute of Pharmacology, Hannover Medical School,

Carl-Neuberg-Straße 1, 30625 Hannover, Germany

e-mail: seifert.roland@mh-hannover.de opposite is true for $\mathrm{Mn}^{2+}$ (Beste et al. 2013) (Table 2). The cation has little impact on $K_{\mathrm{M}}$ of GTP for pGC-A, but for ITP, $\mathrm{Mn}^{2+}$ is preferred. These biochemical activities of sGC and pGC-A were unequivocally documented using highly purified substrates and highly specific and sensitive high-performance liquid chromatography tandem mass spectrometry (HPLCMS/MS) methods (Beste et al. 2012, 2013).

However, the crucial question is whether the cIMP-forming activities of GCs observed in vitro also occur in intact cells. A recent review expressed skepticism that cIMP functions as an independent second messenger, and that it has yet to be unequivocally demonstrated that cIMP exists in intact cells and/or tissues (Beste and Seifert 2013). Previous claims regarding the assumed existence of cIMP in organs are most likely unsubstantiated due to insufficient sensitivity of the primordial MS methods employed (Newton et al. 1986). There were no follow-up studies addressing the claims of the Newton group until very recently. In subsequent studies with the most advanced HPLC-MS/MS techniques currently available, we have shown that HEK293 cells possess very high basal concentrations of adenosine 3',5'-cyclic monophosphate (cAMP), cytidine 3',5'-cyclic monophosphate (cCMP), and uridine $3^{\prime}, 5^{\prime}$-cyclic monophosphate (cUMP) that are controlled by the soluble adenylyl cyclase (sAC) (Hasan et al. 2014). However, cIMP levels were far below detection limit (Beste et al. 2013). A straightforward explanation for this negative finding is that cellular ITP concentrations are very low so that there is no ITP substrate for nucleotidyl cyclase to act upon. In accordance with this hypothesis, sophisticated HPLC-MS/MS analysis of neuronal cells revealed that only inosine 5'-monophosphate (IMP) is present at low levels, but no ITP (Göttle et al. 2013). The most important review in the field of cellular purine nucleotide concentrations (Traut 1994) also mentions just IMP as physiological cell constituent. Traut (1994) does not even depict ITP in his scheme of purine metabolism. Of course, it cannot be excluded that 


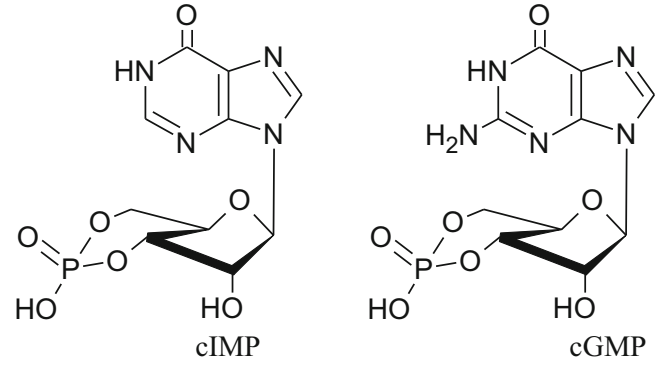

Fig. 1 Structures of cIMP and cGMP

pathophysiological conditions such as hypoxia increase cellular ITP levels (Chen et al. 2014), but common sense would rather expect a decrease of the levels of all nucleoside $5^{\prime}$ triphosphates. We did not explicitly analyze the effect of specific pathophysiological conditions on cNMP levels in intact cells, but another type of experimental cell stress, i.e. the process of cell transfection with exogenous plasmid DNA, substantially decreases basal cNMP levels (Bähre et al. 2014).

On this biochemical background, a recent study by Chen et al. (2014) merits discussion. These authors suggest that under conditions of hypoxia, sGC produces cIMP, but not cGMP in coronary arteries and by such a mechanism, cIMP supposedly induces vasoconstriction instead of the canonical cGMP-dependent vasodilation. The authors also suggest that at least part of the vasoconstriction is mediated via activation of rho kinase. This proposal explicitly implies that cIMP must activate a different effector than PKG. The reduction of affinity of PKG for cIMP compared to cGMP by two orders of magnitude could be compatible with the assumption that cIMP bypasses this kinase (Wolter et al. 2011) (Table 1), but what could then be the target of cIMP? Another straightforward candidate for cIMP could be cAMP-dependent protein kinase (PKA). In fact, cIMP activates two isoforms of PKA (RI $\alpha$ and RII $\alpha$ ) with about tenfold higher potency than cGMP (Wolter et al. 2011) (Table 1). However, an activation of PKA would also be expected to result in vasodilation, but not vasoconstriction (Pelligrino and Wang 1998).

Therefore, one would have to postulate a novel cIMPspecific effector protein. Such a protein would have to exhibit much higher affinity for cIMP than for cGMP, i.e., at least three orders of magnitude, in order to account for the opposing actions of cGMP and cIMP and eliminate any cross-talk of

Table $1 \mathrm{pEC}_{50}$ values of cIMP and cGMP for activation of PKGI $\alpha$, PKA RI $\alpha$, and PKA RII $\alpha$

\begin{tabular}{lll}
\hline $\mathrm{pEC}_{50}$ & cIMP & cGMP \\
\hline PKGI $\alpha$ & 4.72 & 6.98 \\
PKA RI $\alpha$ & 5.89 & 4.91 \\
PKA RII $\alpha$ & 5.37 & 4.51 \\
\hline
\end{tabular}

Data were taken from Wolter et al. (2011)
cIMP with PKG and PKA. To this end, such an effector protein has not yet been identified to the best of my knowledge. Purified human phosphodiesterases are very promiscuous with respect to hydrolysis of cGMP and cIMP (Reinecke et al. 2011). However, it is noteworthy that mammalian membranous ACs exhibit $\sim 25$ - to 80 -fold higher affinity for certain $2^{\prime}, 3^{\prime}$-O-ribosyl-substituted hypoxanthine nucleoside $5^{\prime}$-triphosphates as compared to the corresponding adenine nucleotides (Seifert et al. 2011; Seifert and Dove 2012). Thus, there is precedence for the existence of proteins with a preference for the base hypoxanthine relative to adenine, but nonetheless, base-specificity is not exceedingly high. The application of cIMP agarose affinity chromatography may be a feasible approach to identify putative cIMP-specific effector proteins (Hammerschmidt et al. 2012).

One should also keep in mind alternative mechanisms of action of cIMP. Specifically, cIMP may be exported from the cytosol into the extracellular space by organic anion transporter 2 or multidrug resistance proteins 4 and 5 (Cropp et al. 2008; Russel et al. 2008; Keppler 2011). By analogy to the cAMP-adenosine-adenine system (Jackson and Raghvendra 2004), extracellular cIMP may then be hydrolyzed to inosine and hypoxanthine. Inosine and hypoxanthine, in turn, could exert biological effects via adenosine or adenine receptors, respectively (Schiedel et al. 2007; da Rocha Lapa et al. 2012). These two mechanisms of action of cIMP were not addressed in the study by Chen et al. (2014). Taken together, based on the available data, it is premature to conclude that cIMP by itself functions as an independent second messenger with functions opposite to those of cGMP.

Table 2 Kinetic parameters of sGC and pGC-A with ITP and GTP as substrates

\begin{tabular}{lll}
\hline Parameter & ITP & GTP \\
\hline $\mathrm{sGC}, \mathrm{Mg}^{2+}$ & & \\
$\quad K_{\mathrm{M}}(\mu \mathrm{M})$ & 796 & 119 \\
$\quad V_{\max }(\mu \mathrm{mol} / \mathrm{mg} / \mathrm{min})$ & 4.9 & 2.2 \\
$\mathrm{sGC}, \mathrm{Mn}^{2+}$ & & \\
$K_{\mathrm{M} 1}(\mu \mathrm{M})$ & 6.6 & 14.2 \\
$K_{\mathrm{M} 2}(\mu \mathrm{M})$ & 13,200 & 4,820 \\
$V_{\max 1}(\mu \mathrm{mol} / \mathrm{mg} / \mathrm{min})$ & 1.4 & 4.1 \\
$V_{\max 2}(\mu \mathrm{mol} / \mathrm{mg} / \mathrm{min})$ & 3.4 & 13.5 \\
$\mathrm{pGC}-\mathrm{A}, \mathrm{Mg}{ }^{2+}$ & & 272 \\
$K_{\mathrm{M}}(\mu \mathrm{M})$ & 1,260 & 7.3 \\
$V_{\max }(\mathrm{nmol} / \mathrm{mg} / \mathrm{min})$ & 1.0 & \\
$\mathrm{pGC}-\mathrm{A}, \mathrm{Mn}{ }^{2+}$ & & 231 \\
$K_{\mathrm{M}}(\mu \mathrm{M})$ & 232 & 9.8 \\
$V_{\max }(\mathrm{nmol} / \mathrm{mg} / \mathrm{min})$ & 14.4 & \\
\hline
\end{tabular}

Data for sGC were taken from Beste et al. (2012). Data for pGC-A were taken from Beste et al. (2013) 


\section{References}

Akimoto M, Zhang Z, Boulton S, Selvaratnam R, VanSchouwen B, Gloyd M, Accili EA, Lange OF, Melacini G (2014) A mechanism for the auto-inhibition of hyperpolarization-activated cyclic nucleotide-gated $(\mathrm{HCN})$ channel opening and its relief by cAMP. J Biol Chem (in press)

Bähre H, Danker KY, Stasch JP, Kaever V, Seifert R (2014) Nucleotidyl cyclase activity of soluble guanylyl cyclase in intact cells. Biochem Biophys Res Commun 443:1195-1199

Beste KY, Seifert R (2013) cCMP, cUMP, cTMP, cIMP and cXMP as possible second messengers: development of a hypothesis based on studies with soluble guanylyl cyclase $\alpha_{1} \beta_{1}$. Biol Chem 394:261-270

Beste KY, Burhenne H, Kaever V, Stasch JP, Seifert R (2012) Nucleotidyl cyclase activity of soluble guanylyl cyclase $\alpha_{1} \beta_{1}$. Biochemistry 51:194 204

Beste KY, Spangler CM, Burhenne H, Koch KW, Shen Y, Tang WJ, Kaever V, Seifert R (2013) Nucleotidyl cyclase activity of particulate guanylyl cyclase A: comparison with particulate guanylyl cyclase and $\mathrm{E}$ and $\mathrm{F}$, soluble guanylyl Cyclase and bacterial adenylyl cyclases CyaA and edema factor. PLoS One 8:e70223

Chen Z, Zhang X, Ying L, Dou D, Li Y, Bai Y, Liu J, Liu L, Feng H, Yu X, Lung SWS, Vanhoutte PM, Gao Y (2014) Cyclic IMP synthesized by $\mathrm{sGC}$ as a mediator of hypoxic contraction of coronary arteries. Am J Physiol Heart Circ Physiol (in press)

Cropp CD, Komori T, Shima JE, Urban TJ, Yee SW, More SS, Giacomini KM (2008) Organic anion transporter 2 (SLC22A7) is a facilitative transporter of cGMP. Mol Pharmacol 73:1151-1158

da Rocha Lapa F, da Silva MD, de Almeida CD, Santos AR (2012) Antiinflammatory effects of purine nucleosides, adenosine and inosine, in a mouse model of pleurisy: evidence for a role of adenosine A2 receptors. Purinergic Signal 8:693-704

Göttle M, Burhenne H, Sutcliffe D, Jinnah HA (2013) Purine metabolism during neuronal differentiation: the relevance of purine synthesis and recycling. J Neurochem 127:805-818

Hammerschmidt A, Chatterji B, Zeiser J, Schröder A, Genieser HG, Pich A, Kaever V, Schwede F, Wolter S, Seifert R (2012) Binding of regulatory subunits of cyclic AMP-dependent protein kinase to cyclic CMP agarose. PLoS One 7:e39848

Hasan A, Danker KY, Wolter S, Bähre H, Kaever V, Seifert R (2014) Soluble adenylyl cyclase accounts for high basal cCMP and cUMP concentrations in HEK293 and B103 cells. Biochem Biophys Res Commun 448:236-240

Jackson EK, Raghvendra DK (2004) The extracellular cyclic AMPadenosine pathway in renal physiology. Annu Rev Physiol 66: 571-599

Keppler D (2011) Multidrug resistance proteins (MRPs, ABCCs): Importance for pathophysiology and drug therapy. Handb Exp Pharmacol 201:299-323

Levy FO (2013) Cardiac PDEs and crosstalk between cAMP and cGMP signaling pathways in the regulation of contractility. NaunynSchmiedebergs Arch Pharmacol 386:665-670

Newton RP, Kingston EE, Haakeem NA, Salih SG, Beynon JH, Moyse CD (1986) Extraction, purification, identification and metabolism of 3',5'-cyclic UMP, 3',5'-cyclic IMP and 3',5'-cyclic dTMP from rat tissues. Biochem J 236:431-439

Pelligrino DA, Wang Q (1998) Cyclic nucleotide crosstalk and the regulation of cerebral vasodilation. Prog Neurobiol 56:1-18

Reinecke D, Burhenne H, Sandner P, Kaever V, Seifert R (2011) Human cyclic nucleotide phosphodiesterases possess a much broader substrate-specificity than previously appreciated. FEBS Lett 585: 3259-3262

Russel FG, Koenderink JB, Masereeuw R (2008) Multidrug resistance protein 4 (MRP4/ABCC4): a versatile efflux transporter for drugs and signaling molecules. Trends Pharmacol Sci 29:200-207

Schiedel AC, Meyer H, Alsdorf BB, Gorzalka S, Brüssel H, Müller CE (2007) $\left[{ }^{3} \mathrm{H}\right]$ Adenine is a suitable radioligand for the labeling of $G$ protein-coupled adenine receptors but shows high affinity to bacterial contaminations in buffer solutions. Purinergic Signal 3:347-358

Schlossmann J, Schinner E (2012) Naunyn-Schmiedebergs Arch Pharmacol 385:243-252

Seifert R, Beste KY (2012) Allosteric regulation of nucleotidyl cyclases: an emerging pharmacological target. Sci Signal 5:pe37

Seifert R, Dove S (2012) Towards selective inhibitors of adenylyl cyclase toxin from Bordetella pertussis. Trends Microbiol 20: 343-351

Seifert R, Lushington GH, Mou TC, Gille A, Sprang SR (2011) Inhibitors of membranous adenylyl cyclases. Trends Pharmacol Sci 33:64-78

Traut TW (1994) Physiological concentrations of purines and pyrimidines. Mol Cell Biochem 140:1-22

Wolter S, Golombek M, Seifert R (2011) Differential activation of cAMPand cGMP-dependent protein kinases by cyclic purine and pyrimidine nucleotides. Biochem Biophys Res Commun 415:563-566 